\title{
Branched-chain amino acids are linked with iron metabolism
}

\author{
Dietmar Enko ${ }^{1,2}$, Thomas Moro ${ }^{1}$, Sandra Holasek ${ }^{3}$, Andreas Baranyi ${ }^{4}$, Wolfgang J. Schnedl ${ }^{5}$, \\ Sieglinde Zelzer ${ }^{1}$, Harald Mangge ${ }^{1}$, Markus Herrmann ${ }^{1}$, Andreas Meinitzer ${ }^{1}$
}

${ }^{1}$ Clinical Institute of Medical and Chemical Laboratory Diagnostics, Medical University of Graz, Graz, Austria; ${ }^{2}$ Institute of Clinical Chemistry and Laboratory Medicine, General Hospital Hochsteiermark, Leoben, Austria; ${ }^{3}$ Department of Immunology and Pathophysiology, Medical University of Graz, Otto Loewi Research Center, Graz, Austria; ${ }^{4}$ Department of Psychiatry and Psychotherapeutic Medicine, Medical University of Graz, Graz, Austria; ${ }^{5}$ Practice for General Internal Medicine, Bruck/Mur, Austria

Contributions: (I) Conception and design: D Enko, A Meinitzer; (II) Administrative support: S Holasek, H Mangge, M Herrmann; (III) Provision of study materials or patients: None; (IV) Collection and assembly of data: D Enko, WJ Schnedl, S Zelzer, A Meinitzer; (V) Data analysis and interpretation: D Enko, WJ Schnedl, H Mangge, M Herrmann, A Meinitzer; (VI) Manuscript writing: All authors; (VII) Final approval of manuscript: All authors.

Correspondence to: Dietmar Enko, Clinical Institute of Medical and Chemical Laboratory Diagnostics, Medical University of Graz, Auenbruggerplatz 15, A-8036 Graz, Austria. Email: enko.dietmar@gmx.at.

\begin{abstract}
Background: The branched-chain amino acids (BCAAs) valine, leucine and isoleucine are reported to influence erythropoiesis and the human iron status. Large study cohorts encompassing biomarkers of iron metabolism and BCAAs are still lacking.

Methods: We investigated potential interactions between blood concentrations of all three BCAAs valine, leucine and isoleucine and biomarkers of iron metabolism [i.e., hemoglobin ( $\mathrm{Hb})$, mean corpuscular volume $(\mathrm{MCV})$, mean corpuscular hemoglobin $(\mathrm{MCH})$, iron, transferrin, ferritin, transferrin saturation, soluble transferrin receptor (sTfR)] in 430 outpatients referred for a medical health check-up. Linear regression models were performed to assess possible associations between variables.

Results: All three BCAAs were positively correlated with Hb, ferritin and the sTfR (r-values: 0.145-0.382; $\mathrm{P}$ values: $<0.001-0.003)$. The strongest correlation was observed between valine and $\mathrm{Hb}(\mathrm{r}=0.382 ; \mathrm{P}$ value $<0.001)$. Linear regression models showed a statistically significant influence of all three BCAAs on $\mathrm{Hb}$ and ferritin ( $\beta$-coefficients: $0.173-0.351$; all P values: $<0.001)$. Seventeen patients with anemia $(4 \%)$ were found with significantly lower serum BCAA concentrations compared to 413 non-anemic individuals $(\mathrm{P}<0.05)$.

Conclusions: These data indicate a pathophysiological link between the three BCAAs valine, leucine and isoleucine and the human iron indicators $\mathrm{Hb}$ and ferritin. Further studies are needed to clarify the exact causal mechanisms of these findings.
\end{abstract}

Keywords: Anemia; branched-chain amino acids (BCAAs); iron metabolism

Submitted Jan 11, 2020. Accepted for publication Sep 20, 2020.

doi: 10.21037/atm-20-624a

View this article at: http://dx.doi.org/10.21037/atm-20-624a

\section{Introduction}

The proteinogenic branched-chain amino acids (BCAAs) valine, leucine and isoleucine belong to the nine essential amino acids for humans. Dietary BCAAs are usually derived from meat, fish, dairy products and eggs. Unlike the other amino acids, only a minor BCAA fraction is metabolized by the liver after absorption in the gut.
The largest part of dietary BCAAs appears in the blood circulation and can be utilized for new protein synthesis (1). Leucine has the most anabolic effect of BCAAs, which is mediated by activation of the mechanistic target of rapamycin complex 1 (mTORC1) $(1,2)$.

The mTORC1 is a multi-protein complex, which regulates protein synthesis and cell growth through various downstream effectors (3). Recent evidence suggests, that 
BCAAs play multiple roles via this mTORC1 pathway and that a metabolic imbalance of these nutrients is associated with diabetes, cardiovascular diseases, obesity, cancer or mood disorders (4-7). High concentrations of BCAAs were shown to promote oxidative stress, inflammation and the migration of peripheral mononuclear blood cells through mTORC1 activation (8).

Certain studies suggested a possible link between BCAAs, mTORC1 and hematological parameters (9-11). Significant increases in hemoglobin $(\mathrm{Hb})$, serum iron, red blood cell count and hematocrit were observed in a small cohort of 23 athletes after a 90-day administration of a BCAAmixture (9). These data implicate, that BCAAs might also promote erythropoiesis and iron status improvement (9). Since the BCAAs are a limiting factor for protein synthesis and cell growth, a nutritional deficiency of them may also lead to lowered protein biosynthesis of haemoglobin and other indicators of iron metabolism. This could represent one possible pathophysiological link between BCAAs and human iron indicators. Many of these effects are mediated by the mTORC1 pathway (6), which may represent a possible link between BCAAs and human iron metabolism.

Currently, serum ferritin and transferrin saturation remain the widespread biomarkers of the human iron status in clinical practice. Both parameters are known to be affected by acute-phase reactions (12), whereas the soluble transferrin receptor (sTfR), which is inversely correlated with the human body's iron stores, is not influenced by acute or chronic inflammatory illness (12-14). Despite the established associations between elevated serum BCAA levels and various diseases, little is known about possible interactions between BCAAs and iron metabolism. There is still a lack of study designs comprising biomarkers of human iron status and BCAAs in large study cohorts. Considering the central role of BCAAs in metabolic pathways, the hypothesis of this study was, that BCAAs may also interact with the human iron metabolism, which also represents an important part of strong health.

The aim of this study was to investigate potential associations between peripheral blood levels of valine, leucine and isoleucine and indicators of iron metabolism [i.e., $\mathrm{Hb}$, mean corpuscular volume (MCV), mean corpuscular hemoglobin $(\mathrm{MCH})$, iron, transferrin, ferritin, transferrin saturation, sTfR] in a large cohort of outpatients referred for a medical health check-up.

We present the following article in accordance with the Materials Design Analysis reporting checklist (available at http://dx.doi.org/10.21037/atm-20-624a).

\section{Methods}

\section{Patients}

In total, 430 ambulatory individuals, who were referred to the outpatient clinic for iron status assessment, were included in this study. They underwent investigation of iron biomarkers (i.e., $\mathrm{Hb}, \mathrm{MCV}, \mathrm{MCH}$, iron, transferrin, ferritin, transferrin saturation, sTfR) and also the BCAAs (i.e., valine, leucine, isoleucine).

The inclusion criteria were a minimum age of 15 years and an obligatory overnight fasting state of $>12 \mathrm{~h}$. Individuals with the unavailability of all study parameters and iron supplementation within the last 12 months were excluded from the study. The study was conducted in accordance with the Declaration of Helsinki (as revised in 2013). The study was approved by the Ethics Committee of the Johannes Kepler University Linz (Linz, Austria) (No. 1027/2019) and informed consent was taken from all patients.

\section{Laboratory procedures}

Fasting blood samples were collected between 8.00 and 10.00 a.m. Hb (anemia: males, $<13 \mathrm{~g} / \mathrm{dL}$; females, $<12 \mathrm{~g} / \mathrm{dL}$ ) and erythrocyte indices (MCV and $\mathrm{MCH}$ ) were determined

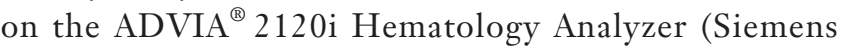
Healthineers, Erlangen, Germany). Ferritin was assessed by chemiluminescent technology, serum iron by photometric method, and transferrin and the sTfR by nephelometric technology on a Dimension Vista ${ }^{\circledR} 1500$ System (Siemens Healthineers, Erlangen, Germany). The percentage of transferrin saturation was calculated based on the formula: transferrin saturation $(\%)=$ serum iron $(\mu \mathrm{g} / \mathrm{dL}) \times 70.9 /$ transferrin $(\mathrm{mg} / \mathrm{dL})$. The reference ranges of the study parameters are listed in Table 1. Iron deficiency was defined as a serum ferritin level $<30 \mu \mathrm{g} / \mathrm{L}$ and/or a transferrin saturation $<20 \%(12,14,15)$.

Serum concentrations of the BCAAs valine, leucine and isoleucine were measured by previously described chromatographic methods $(5,16)$. Briefly, after precipitation of serum with perchloric acid following neutralization of the supernatant with sodium carbonate, the extracted amino acids were derivatized with o-phtalaldehyde and separated on a reversed phase column with gradient elution. Quantification was performed with ratios of fluorescence signals of the interesting amino acids to the internal standard norvaline in comparison to the appropriated calibration curves. Intra- and inter-assay correlation coefficients were all $<10 \%$. The reference ranges of BCAAs 
Table 1 Baseline characteristics of the study population

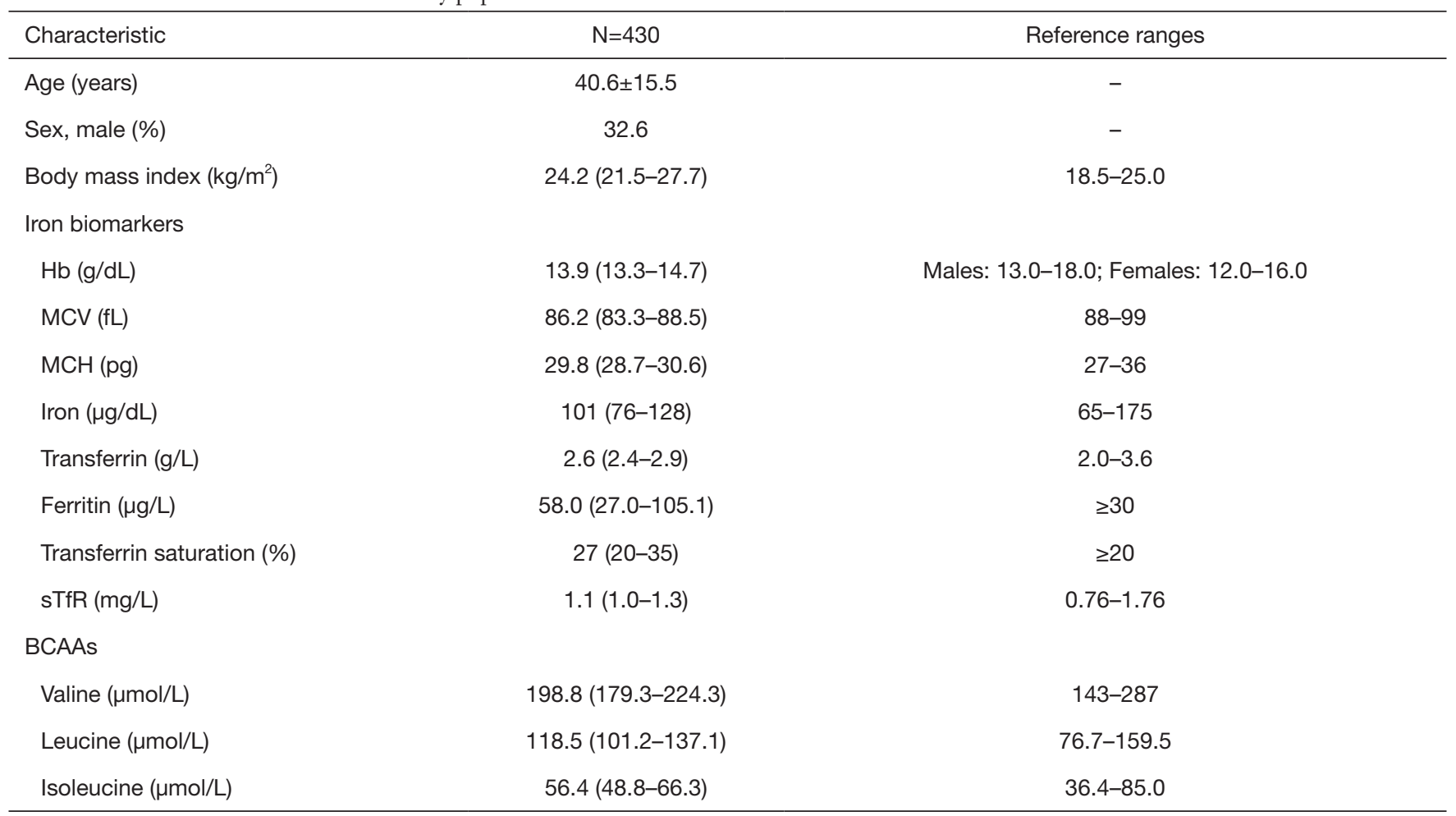

Data are presented as means \pm standard deviation, or medians (Q1-Q3), or percentage. Hb, hemoglobin; MCV, mean corpuscular volume; $\mathrm{MCH}$, mean corpuscular hemoglobin; sTfR, soluble transferrin receptor; BCAAs, branched-chain amino acids.

were calculated according to the literature (17).

\section{Statistical analysis}

Sample size estimation was performed according to the literature (18). A sample size of approximately 400 individuals was assessed to obtain insights about potential associations between BCAAs and indicators of iron metabolism.

The distribution of the data was calculated with the Kolmogorov-Smirnov test. Not normally distributed continuous variables were presented as medians with interquartile ranges $(\mathrm{Q} 1-\mathrm{Q} 3)$. Categorical variables were expressed as percentages. To evaluate potential correlation between two continuous variables the Spearman's rank correlation coefficient (r) was used. Furthermore, linear regression models were performed to assess the association between variables. The Mann-Whitney $U$ test was calculated for the comparison between two groups for not normally distributed continuous variables. A P value $<0.05$ was considered statistically significant. For statistical analysis, the Analyse-it ${ }^{\circledR}$ software version 4.92 (Analyse-it Software, Ltd., Leeds, United Kingdom) was used.

\section{Results}

\section{Study population characteristics}

The baseline characteristics of the study population are shown in Table 1. The average age was $40.6 \pm 15.5$ years, and $32.6 \%$ of participants were men. Based on a serum $\mathrm{Hb}$-level $<13 \mathrm{~g} / \mathrm{dL}$ for men and $<12 \mathrm{~g} / \mathrm{dL}$ for women, 17 ( 2 men and 15 women) out of 430 individuals (4.0\%) were found with anemia. Based on a ferritin of $<30 \mu \mathrm{g} / \mathrm{L}$ and/or a transferrin saturation of $<20 \%, 159$ (20 men and 139 women) patients $(37.0 \%)$ were identified with iron deficiency.

\section{Correlations between BCAAs and parameters of iron metabolism}

As illustrated in Table 2, the BCAAs valine, leucine and isoleucine were significantly correlated with $\mathrm{Hb}$, ferritin and the sTfR. The strongest positive correlations were observed between BCAAs and $\mathrm{Hb}$ (r-values: 0.244-0.382; all $P$ values $<0.001$ ), followed by BCAAs and ferritin (r-values: $0.182-0.312$; all $\mathrm{P}$ values $<0.001$ ), and BCAAs and sTfR (r-values: $0.145-0.157$; P values: $0.001-0.003$ ), respectively. 
Table 2 Spearman's rank correlation coefficients (r) between BCAAs, Hb, ferritin, and sTfR

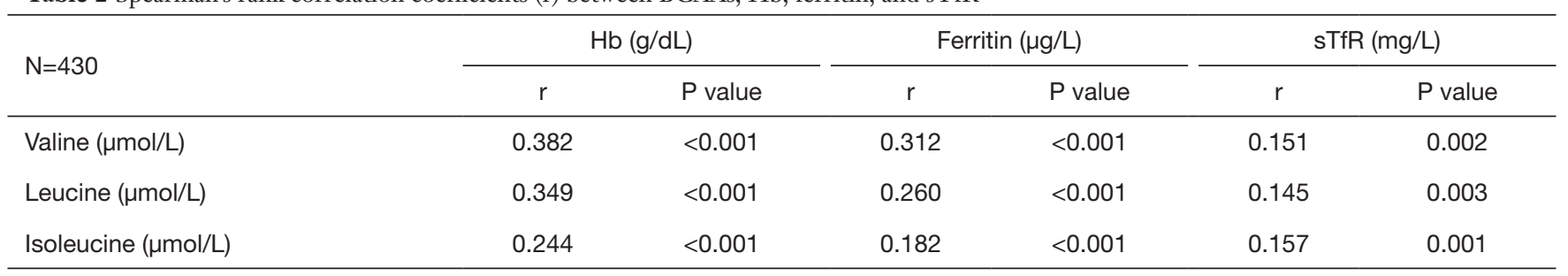

BCAAs, branched-chain amino acids; $\mathrm{Hb}$, hemoglobin; sTfR, soluble transferrin receptor.

Table 3 Univariate linear regression models for $\mathrm{Hb}$, ferritin and sTfR

\begin{tabular}{|c|c|c|c|c|c|c|}
\hline $\mathrm{N}=430$ & \multicolumn{2}{|c|}{$\mathrm{Hb}(\mathrm{g} / \mathrm{dL})$} & \multicolumn{2}{|c|}{ Ferritin $(\mu \mathrm{g} / \mathrm{L})$} & \multicolumn{2}{|c|}{$\mathrm{sTfR}(\mathrm{mg} / \mathrm{L})$} \\
\hline Valine $(\mu \mathrm{mol} / \mathrm{L})$ & 0.351 & $<0.001$ & 0.307 & $<0.001$ & 0.084 & 0.083 \\
\hline Leucine $(\mu \mathrm{mol} / \mathrm{L})$ & 0.331 & $<0.001$ & 0.254 & $<0.001$ & 0.077 & 0.112 \\
\hline Isoleucine ( $\mu \mathrm{mol} / \mathrm{L})$ & 0.195 & $<0.001$ & 0.173 & $<0.001$ & 0.093 & 0.055 \\
\hline
\end{tabular}

$\mathrm{Hb}$ hemoglobin, sTfR soluble transferrin receptor.

\section{Associations of $H b$, ferritin and sTfR with BCAAs}

The univariate linear regression models for $\mathrm{Hb}$, ferritin and sTfR are presented in Table 3. The BCAAs valine, leucine and isoleucine showed a statistically significant influence on $\mathrm{Hb}$ and ferritin ( $\beta$-coefficients: 0.173-0.351; all $\mathrm{P}$ values $<0.001)$. Valine and isoleucine were in tendency ( $\beta$-coefficients: 0.084 and 0.093 ; $P$ values: 0.055 and 0.083 ) associated with sTfR. Figure 1 demonstrates linear regression models between $\mathrm{Hb}$, ferritin, sTfR and all three BCAAs (valine + leucine + isoleucine) taken together.

As shown in Figure 2, patients with anemia had significantly lower median serum BCAA (valine + leucine + isoleucine) levels [329.1 (292.0-393.0) vs. 373.6 (335.6427.9) $\mu \mathrm{mol} / \mathrm{L}, \mathrm{P}<0.05]$ compared to individuals without anemia.

\section{Discussion}

In the current study, the three BCAAs valine, leucine and isoleucine were significantly correlated with $\mathrm{Hb}$, ferritin and the $\mathrm{sTfR}$. The linear regression models showed strongest associations between BCAAs and Hb. Lowered BCAA levels were found with lowered $\mathrm{Hb}$ values.

These findings are consistent with the results of a previous study by Chung et al., who demonstrated, that the uptake of the essential BCAAs is a very important component of $\mathrm{Hb}$ synthesis in hematopoietic stem cells (19). In this context, the mTORC1 was shown to be a key regulator in hematopoietic stem cell function (20). This multi-protein complex acts as an intracellular sensor of BCAA availability, which coordinates the $\mathrm{Hb}$ production $(19,21)$.

Here, the strongest correlation was found between the BCAA valine and $\mathrm{Hb}(\mathrm{r}=0.382 ; \mathrm{P}<0.001)$. These data corroborate a recently published experimental study, which reported, that hematopoietic stem cells are highly sensitive to valine availability (22). Moreover, proteinenergy malnutrition in fasting surgical patients showed, that low valine blood levels were highly correlated with low $\mathrm{Hb}$ concentrations $(r=0.52 ; \mathrm{P}<0.05)(23)$. This fact suggests that insufficient protein intake may be one possible reason for BCAA depletion and $\mathrm{Hb}$ reduction (23).

In the present study, patients with anemia were found with significantly lower serum BCAA concentrations compared to individuals without anemia. Several recent studies demonstrated that treatment with the BCAA leucine improves the anemia of the Diamond-Blackfan and of the 5q-myelodysplastic syndrome (24-27). Haploinsufficiency of ribosomal proteins in hematopoietic stem cells is the proposed cause of the anemia observed in these disorders $(24,28)$. Leucine activates the mTORC1 pathway, which may overcome the ribosomal protein deficiencies and 

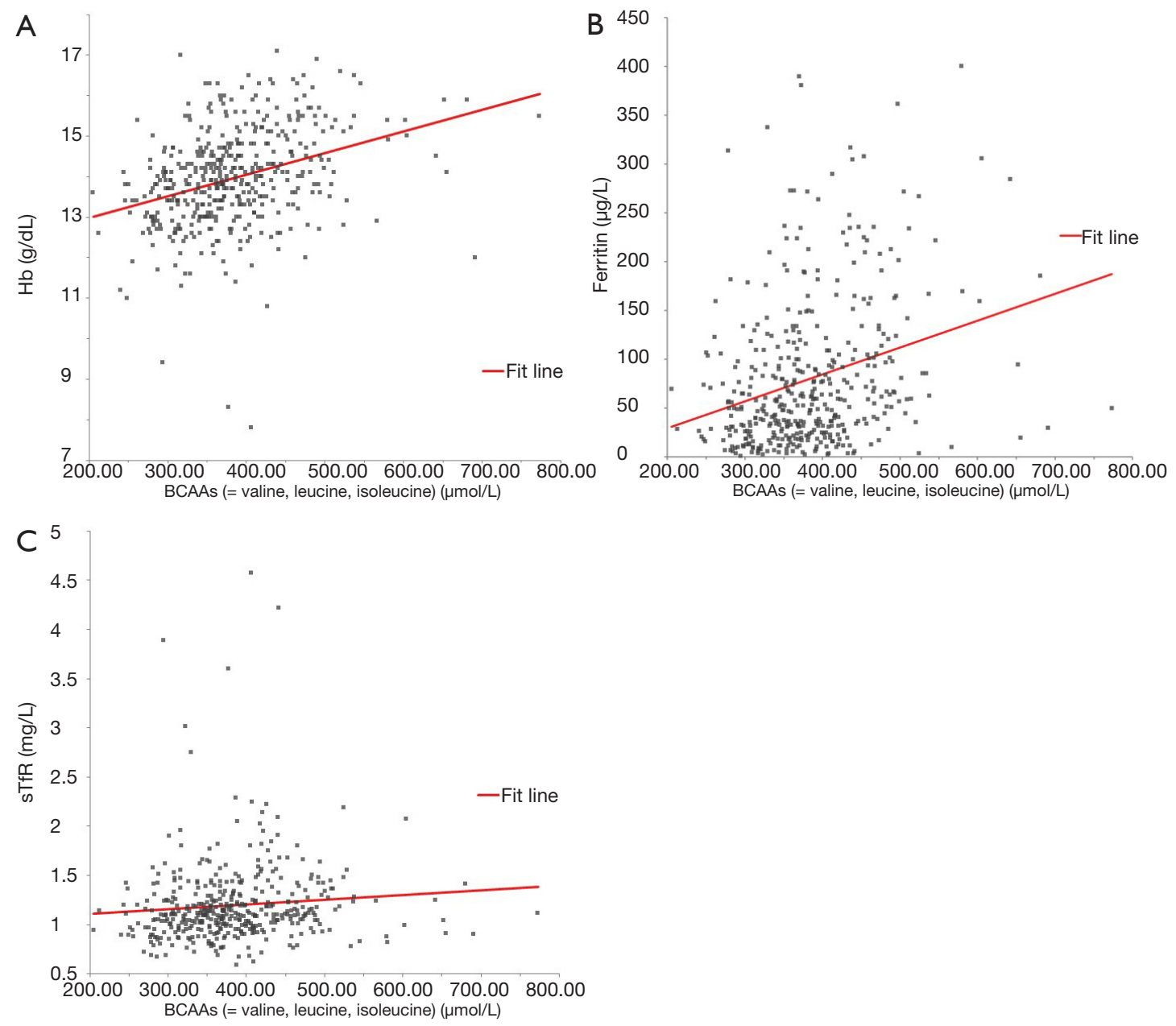

Figure 1 Univariate linear regression models between parameters of iron metabolism and BCAAs (valine, leucine, isoleucine). (A) Hb vs. BCAAs $(\beta$-coefficient $=0.341, \mathrm{P}<0.001)$, (B) ferritin $v$ s. BCAAs $(\beta$-coefficient $=0.286, \mathrm{P}<0.001)$ and $(\mathrm{C})$ sTfR $v$ s. BCAAs $(\beta$-coefficient $=0.091$, $\mathrm{P}=0.061)$. BCAAs, branched-chain amino acids; Hb, hemoglobin; sTfR, soluble transferrin receptor.

rescue the anemia $(24,29)$. Recently, in elderly patients with myelodysplastic syndrome, oral leucine administration was shown to increase the absolute reticulocyte count, which is an indicator of hematopoietic functionality (30).

Present data show, that the valine, leucine and isoleucine blood levels significantly correlated with the iron storage protein ferritin. Lowered BCAA levels were found with lowered ferritin values. In a previous published study, it was shown that apoferritin, which is the hollow shell protein component of ferritin, contains amino acid residues of all three BCAAs (31). Little correlation was also observed between sTfR and BCAAs. Previous determination of the nucleotide sequence of human sTfR showed that the amino acid sequence of this iron biomarker comprises all three
BCAAs (32).

Valine, leucine and isoleucine are cellular nutrients, which are essential for the synthesis of human structural and functional proteins. A previous report demonstrated an increasement of $\mathrm{Hb}$, ferritin, red blood cell count and hematocrit in training athletes after daily consumption of an amino acid mixture, which encompassed all three BCAAs (33). Intermediaries of BCAA metabolism are also known to induce fetal $\mathrm{Hb}$ during definitive erythropoiesis (34).

The impact of BCAAs on erythropoiesis and iron metabolism should be investigated in further longitudinal studies, which are able to clarify the exact pathophysiological mechanisms of this observed link. The major advance of the present study is that the BCAAs and iron biomarkers were 


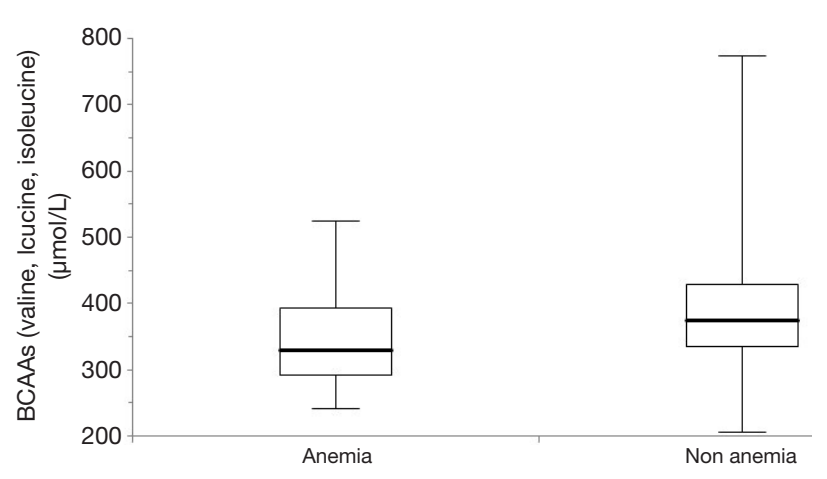

Figure 2 BCAAs (valine, leucine, isoleucine) and anemia. Boxand-whisker plot of BCAA serum level comparison between 17 anemic and 413 non-anemic individuals $(\mathrm{P}=0.046)$. The central boxes represent the 25 th to 75 th percentile range. The lines inside the boxes show the median value for each group. Minimum and maximum are indicated as whiskers with end caps. BCAAs, branched-chain amino acids.

measured in a well characterized large study cohort.

The major limitation of this cross-sectional study is that lifestyle factors and dietary habits were not considered.

\section{Conclusions}

Significant associations between the BCAAs valine, leucine, and isoleucine and the iron biomarkers $\mathrm{Hb}$ and ferritin were observed. Patients with anemia showed significantly lower serum BCAA levels compared to individuals without anemia. Lowered BCAA concentrations were found with lowered ferritin levels. These findings suggest a pathophysiological link between BCAA and iron metabolism.

\section{Acknowledgments}

Funding: None.

\section{Footnote}

Reporting Checklist: The authors have completed the MDAR reporting checklist. Available at http://dx.doi.org/10.21037/ atm-20-624a

Data Sharing Statement: Available at http://dx.doi. org/10.21037/atm-20-624a
Conflicts of Interest: All authors have completed the ICMJE uniform disclosure form (Available at http://dx.doi. org/10.21037/atm-20-624a). The authors have no conflicts of interest to declare.

Ethical Statement: The authors are accountable for all aspects of the work in ensuring that questions related to the accuracy or integrity of any part of the work are appropriately investigated and resolved. The study was conducted in accordance with the Declaration of Helsinki (as revised in 2013). The study was approved by the Ethics Committee of the Johannes Kepler University Linz (Linz, Austria) (No. 1027/2019) and informed consent was taken from all patients. This study can provide new insights in the pathophysiological link between BCAAs and iron metabolism.

Open Access Statement: This is an Open Access article distributed in accordance with the Creative Commons Attribution-NonCommercial-NoDerivs 4.0 International License (CC BY-NC-ND 4.0), which permits the noncommercial replication and distribution of the article with the strict proviso that no changes or edits are made and the original work is properly cited (including links to both the formal publication through the relevant DOI and the license). See: https://creativecommons.org/licenses/by-nc-nd/4.0/.

\section{References}

1. Neinast $M$, Murashige D, Arany Z. Branched chain amino acids. Annu Rev Physiol 2019;81:139-64.

2. Saxton RA, Knockenhauer KE, Wolfson RL, et al. Structural basis for leucine sensing by the Sestrin2mTORC1 pathway. Science 2016;351:53-8.

3. Laplante M, Sabatini DM. mTOR signaling at a glance. J Cell Sci 2009;122:3589-94.

4. Zhou M, Shao J, Wu CY, et al. Targeting BCAA catabolism to treat obesity-associated insulin resistance. Diabetes 2019;68:1730-46.

5. Mangge $\mathrm{H}$, Zelzer S, Prüller F, et al. Branched-chain amino acids are associated with cardiometabolic risk profiles found already in lean, overweight and obese young. J Nutr Biochem 2016;32:123-7.

6. Nie $\mathrm{C}, \mathrm{He} \mathrm{T}$, Zhang $\mathrm{W}$, et al. Branched chain amino acids: beyond nutrition metabolism. Int J Mol Sci 2018;19:E954.

7. Baranyi A, Amouzadeh-Ghadikolai O, von Lewinski D, 
et al. Branched-chain amino acids as new biomarkers of major depression - a novel neurobiology of mood disorder. PLoS One 2016;11:e0160542.

8. Zhenyukh O, Civantos E, Ruiz-Ortega M, et al. High concentration of branched-chain amino acids promotes oxidative stress, inflammation and migration of human peripheral blood mononuclear cells via mTORC1 activation. Free Radic Biol Med 2017;104:165-77.

9. Ohtani M, Maruyama K, Sugita M, et al. Amino acid supplementation affects hematological and biochemical parameters in elite rugby players. Biosci Biotechnol Biochem 2001;65:1970-6.

10. Diekmann F, Rovira J, Diaz-Ricart M, et al. mTOR inhibition and erythropoiesis: microcytosis or anaemia? Nephrol Dial Transplant 2012;27:537-41.

11. Kim Y, Men SS, Liang C, et al. Effects of long-term exposures to low iron and branched-chain amino acid containing diets on aging skeletal muscle of Fisher 344 Brown Norway rats. Appl Physiol Nutr Metab 2018;43:165-73.

12. Enko D, Wagner H, Kriegshäuser G, et al. Assessment of human iron status: A cross-sectional study comparing the clinical utility of different laboratory biomarkers and definitions of iron deficiency in daily practice. Clin Biochem 2015;48:891-6.

13. Mast AE, Blinder MA, Gronowski AM, et al. Clinical utility of the soluble transferrin receptor and comparison with serum ferritin in several populations. Clin Chem 1998;44:45-51.

14. Muñoz M, Villar I, García-Erce JA. An update on iron physiology. World J Gastroenterol 2009;15:4617-26.

15. Enko D, Wagner H, Kriegshäuser G, et al. Iron status determination in individuals with Helicobacter pylori infection: conventional vs. new laboratory biomarkers. Clin Chem Lab Med 2019;57:982-9.

16. Schwarz EL, Roberts WL, Pasquali M. Analysis of plasma amino acids by HPLC with photodiode array and fluorescence detection. Clin Chim Acta 2005;354:83-90.

17. Yamamoto H, Kondo K, Tanaka T, et al. Reference intervals for plasma-free amino acid in a Japanese population. Ann Clin Biochem 2016;53:357-64.

18. Eng J. Sample size estimation: How many individuals should be studied? Radiology 2003;227:309-13.

19. Chung J, Bauer DE, Ghamari A, et al. The mTORC1/4EBP pathway coordinates hemoglobin production with L-leucine availability. Sci Signal 2015;8:ra34.
20. Kalaitzidis D, Sykes SM, Wang Z, et al. mTOR complex 1 plays critical roles in hematopoiesis and Pten-loss-evoked leukemogenesis. Cell Stem Cell 2012;11:429-39.

21. Nathan DG. Amino acid uptake in erythropoiesis. Sci Signal 2015;8:fs9.

22. Wilkinson AC, Morita M, Nakauchi H, et al. Branchedchain amino acid depletion conditions bone marrow for hematopoietic stem cell transplantation avoiding amino acid imbalance-associated toxicity. Exp Hematol 2018;63:12-6.e1.

23. Young GA, Hill GL. Evaluation of protein-energy malnutrition in surgical patients from plasma valine and other amino acids, proteins, and anthropometric measurements. Am J Clin Nutr 1981;34:166-72.

24. Payne EM, Virgilio M, Narla A, et al. L-leucine improves the anemia and developmental defects associated with Diamond-Blackfan anemia and $\operatorname{del}(5 \mathrm{q})$ MDS by activating the mTOR pathway. Blood 2012;120:2214-24.

25. Jaako P, Debnath S, Olsson K, et al. Dietary L-leucine improves the anemia in a mouse model for DiamondBlackfan anemia. Blood 2012;120:2225-8.

26. Narla A, Payne EM, Abayasekara N, et al. L-leucine improves the anaemia in models of Diamond Blackfan anaemia and the $5 \mathrm{q}$ - syndrome in a TP53-independent way. Br J Haematol 2014;167:524-8.

27. Pospisilova D, Cmejlova J, Hak J, et al. Successful treatment of a Diamond-Blackfan anemia patient with amino acid leucine. Haematologica 2007;92:e66-7.

28. Pereboom TC, Bondt A, Pallaki P, et al. Translation of branched-chain aminotransferase-1 transcripts is impaired in cells haploinsufficient for ribosomal protein genes. Exp Hematol 2014;42:394-403.e4.

29. Yip BH, Pellagatti A, Vuppusetty C, et al. Effects of L-leucine in 5q-syndrome and other RPS14-deficient erythroblasts. Leukemia 2012;26:2154-8.

30. Ito K, Hayashi T, Inaguma Y, et al. Effect of L-leucine therapy on hematopoietic function in elderly myelodysplastic syndrome patients. Biol Pharm Bull 2019;42:1651-7.

31. Addison JM, Fitton JE, Lewis WG, et al. The amino acid sequence of human liver apoferritin. FEBS Lett 1983;164:139-44.

32. Schneider C, Owen MJ, Banville D, et al. Primary structure of human transferrin receptor deduced from the mRNA sequence. Nature 1984;311:675-8.

33. Ohtani M, Maruyama K, Suzuki S, et al. Changes in 
hematological parameters of athletes after receiving daily dose of a mixture of 12 amino acids for one month during the middle- and long-distance running training. Biosci Biotechnol Biochem 2001;65:348-55.

34. Karkashon S, Raghupathy R, Bhatia H, et al.

Cite this article as: Enko D, Moro T, Holasek S, Baranyi A, Schnedl WJ, Zelzer S, Mangge H, Herrmann M, Meinitzer A. Branched-chain amino acids are linked with iron metabolism. Ann Transl Med 2020;8(23):1569. doi: 10.21037/atm-20-624a
Intermediaries of branched chain amino acid metabolism induce fetal hemoglobin, and repress SOX6 and BCL11A, in definitive erythroid cells. Blood Cells Mol Dis 2015;55:161-7. 